\title{
Strates
}

STRATES Matériaux pour la recherche en sciences sociales

13 | 2007

Paysage urbain: genèse, représentations, enjeux contemporains

\section{Le paradoxe du paysage urbain dans les discours paysagistes}

\section{Florent Hebert}

\section{OpenEdition}

Journals

Édition électronique

URL : http://journals.openedition.org/strates/5493

DOI : $10.4000 /$ strates.5493

ISSN : $1777-5442$

Éditeur

Laboratoire Ladyss

Édition imprimée

Date de publication : 31 décembre 2007

ISSN : 0768-8067

Référence électronique

Florent Hebert, «Le paradoxe du paysage urbain dans les discours paysagistes », Strates [En ligne],

13 | 2007, mis en ligne le 22 octobre 2008, consulté le 21 décembre 2020. URL : http://

journals.openedition.org/strates/5493; DOI : https://doi.org/10.4000/strates.5493

Ce document a été généré automatiquement le 21 décembre 2020.

Tous droits réservés 


\title{
Le paradoxe du paysage urbain dans les discours paysagistes
}

\author{
Florent Hebert
}

Je déteste la périphérie de Lyon où je vis. Pour

rejoindre la faculté des sciences où j'étudie, je

repère une succession de chemins de traverse qui

conduit jusqu'au campus en longeant les bras

morts du Rhône. De lônes en terrains vagues, souvent piégé par des culs-de-sac, je recompose au fil des mois une sorte de paysage continu qui

me rend cette banlieue supportable.

(Michel Desvigne, architecte paysagiste ${ }^{1}$ )

L'expression "paysage urbain » est aujourd'hui employée de façon récurrente et un peu passe-partout (notamment dans le domaine de l'aménagement). Les architectes paysagistes semblent toutefois faire singulièrement exception, lorsqu'ils parlent de leurs réalisations ou de leurs projets en cours (et qui concernent parfois, avec Michel Desvigne, de larges pans de ville); il est très rare alors qu'un paysage soit qualifié d'urbain, même si les interventions qui vont tendre à en révéler la substance intéressent la ville et ses quartiers. C'est cette « abstinence » relative mais surprenante qui va être discutée ici, prétexte à poser certaines hypothèses sur les possibilités d'une «mise en paysage» des espaces urbains. Il ne s'agit pas de porter une quelconque critique à l'encontre de ces pratiques, qui s'inscrivent dans des logiques professionnelles et opérationnelles tout à fait pertinentes; il s'agit plutôt de s'interroger sur la valeur paradigmatique du paysage dans la production des espaces urbains, et sur sa valeur sociale en tant qu'expérience quotidienne. La complexité revendiquée du projet de paysage légitime effectivement la prétention des architectes paysagistes à intervenir dans des villes aux enjeux et aux imbrications spatiales devenus complexes - mais provoquent-ils pour autant l'apparition de réels « paysages urbains »? Cette apparente contradiction s'avère révélatrice des manières de faire la ville, à travers les manières de voir de ceux qui la font, en matière de paysage notamment. 
2 Depuis une trentaine d'années, un champ de réflexion théorique sur « le » paysage s'est institué en France; cet effort a porté notamment sur la valorisation culturelle et esthétique des représentations paysagistes et paysagères ${ }^{2}$, d'un point de vue aussi bien artistique que social ${ }^{3}$. Il s'est agi notamment d'expliciter les différences entre l'approche environnementale et l'approche paysagère. Ainsi le philosophe Alain Roger n'hésite pas à affirmer que «le paysage, quant à lui, est une notion plus ancienne, d'origine artistique [...], et relevant, comme telle, d'une analyse essentiellement esthétique »; et "toute l'histoire du paysage occidental, aussi bien qu'extrêmeoriental, le montre à l'évidence : le paysage est d'abord le produit d'une opération perceptive, c'est-à-dire une détermination socioculturelle ${ }^{4} »$. C'est essentiellement de ce point de vue que la notion de paysage sera comprise ici, et discutée ; elle souscrit à l'emploi usuel du mot paysage en France, c'est-à-dire non seulement cette "partie d'un pays que la nature présente à un observateur ${ }^{5}$ ", mais également certain modèle pictural auquel cet observateur se réfère immanquablement ${ }^{6}$.

3 Implicitement, cet « objet » paysage renvoie la plupart du temps à des modèles que l'on qualifiera ici de "naturels", en tant qu'ils impliquent par le regard une relation sensible entre la société humaine et les autres formes de vie qu'elle organise, ne seraitce qu'en leur donnant une valeur esthétique (la relation au monde rural est donc particulièrement mise en avant, et l'art des jardins relève du même souci d'organiser le naturel). Le travail original entrepris en France semble avoir construit ses références sur le postulat d'une telle organisation sensible de la nature. La "théorie du paysage » aura ainsi largement délaissé l'analyse de l'espace urbain (sans toutefois ignorer celuici), contrairement à ce qui fut entrepris dans le même temps (et dans ce même domaine de la représentation) par d'autres disciplines ${ }^{7}$. Dans ces conditions, il convient de lever l'ambiguïté de l'existence possible de "paysages urbains», au regard de cet apport théorique sur le paysage, qui dépasse implicitement la sensibilité conventionnelle aux choses de la nature.

4 Il faudra pour cela partir de l'hypothèse que les représentations sociales et esthétiques de l'urbain s'opposent finalement à celles d'une nature toujours plus utopique et toujours plus désirable (ces dernières faisant, seules, "paysage »). Il sera donc recherché ici non pas tant les traces de contradictions dans le discours des théoriciens et des praticiens qui leur font écho, mais plutôt celles d'une construction complexe et paradoxale des espaces urbains (par leur mise en relation à des espaces décrits comme naturels).

5 A. Roger soutient ainsi le principe qu'une relation esthétique critique peut donner naissance, par sa seule diffusion, à de nouveaux modèles de paysages. Il conviendra donc de s'interroger : non pas tant sur ce que sont ou ce que devraient être les paysages de l'urbain - sont-ils ou non des modèles de "paysages »? - mais plutôt sur la façon dont les représentations de l'espace sont convoquées à travers le projet de paysage, puis utilisées implicitement dans la fabrication plus ou moins ordonnée du milieu urbain (désir d'invoquer le naturel comme ce qui donne sens à la surprésence humaine, répulsion à envisager ne pas pouvoir le faire). Or s'il est indéniable que l'image des villes se trouve ainsi modifiée matériellement et immatériellement par les pratiques paysagistes, ces interventions autorisent-elles pour autant à parler du paysage comme d'un mode de représentation pertinent du milieu urbain ${ }^{8}$ ?

6 Car les représentations culturelles de ce dernier s'opposent (en apparence) à celles du milieu naturel; en évoquant par le projet paysagiste la possibilité (le désir) d'un 
paysage utopique, il s'agit d'abord de rendre la ville moins repoussante. Les paysagistes tendent ainsi à " naturaliser » l'espace urbain (car « le paysage s'identifie à la nature ${ }^{9}$ ") en le rapportant (visuellement, symboliquement ou stratégiquement) à des échelles spatiales qui excèdent la simple localisation des lieux à aménager. D'où l'importance à mieux comprendre le rôle que joue dans le projet de paysage la reconnaissance du site d'intervention : car c'est à ce moment que s'établit (en réaction souvent à la répulsion qu'exerce le milieu urbain) le premier désir de paysage, qui conditionne la réalisation du projet. Le travail de la philosophe Anne Cauquelin ${ }^{10}$ se révèlera précieux à ce stade, car il y est fait état de rapports d'ordre spatial entre paysage et site, notions toutes deux abondamment utilisées par les architectes paysagistes.

Aménagement urbain et paysage

Un paysagiste, c'est quelqu'un qui transforme un projet d'aménagement en projet de paysage. ${ }^{11}$

7 En sélectionnant quelques numéros récents de revues européennes, liées à l'aménagement urbain et portant explicitement sur des problématiques paysagères ${ }^{12}$, on s'aperçoit bien qu'il est difficile pour les paysagistes français de parler explicitement de "paysages urbains » - que ceux-ci soient décrits comme étant déjà constitués, ou bien en devenir. L'expression est par contre beaucoup plus courante dans les textes qui commentent les projets de paysage, d'architecture ou d'urbanisme : articles spécialisés ou grand public; numéros spéciaux ou thématiques de revues; mot clef employé dans le référencement des centres de documentation ${ }^{13}$. On remarque d'ailleurs à travers ces commentaires que l'action des paysagistes a pris en France (depuis une quinzaine d'années) une place essentielle dans la définition des pratiques de production de l'espace urbain: Alexandre Chemetoff, puis Michel Corajoud, figures émérites du paysagisme, ont ainsi reçu en 2000 et 2003 le Grand prix de l'urbanisme décerné par le ministère de l'Équipement.

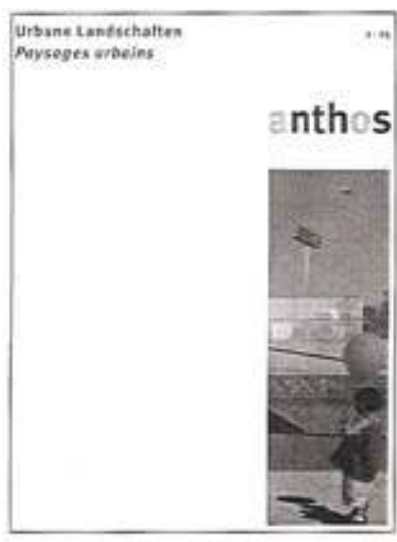

Anthos 0 DR

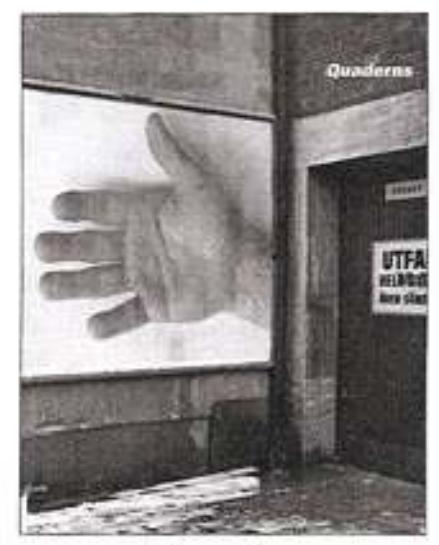

Quoderns $\%$ DR

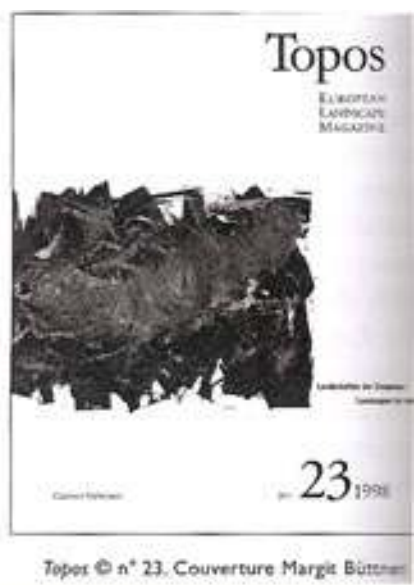

8 De fait, c'est généralement dans le cadre d'une réflexion des paysagistes sur l'aménagement urbain que le paysage « urbain » a le plus de chance d'être nommé, à défaut d'être convoqué ${ }^{14}$. L'expression semble alors utilisée pour caractériser les ambiances (existantes ou à venir) de certaines parties de villes. C'est donc une utilisation essentiellement descriptive qui est faite de la représentation paysagère, consentie au titre d'une certaine cohérence (voire d'une réelle qualité pittoresque) d'une rue ou d'un quartier. À cette échelle, les interventions spatiales (auxquelles peuvent prétendre non seulement les paysagistes, mais surtout les architectes et les plasticiens) peuvent donc s'en tenir à un cadre spatial relativement restreint. D'où cette 
distinction assez nette qu'un paysagiste comme Jacques Coulon revendique entre un projet d'aménagement et un projet de paysage. Cette opposition mériterait sans doute d'être étudiée plus en profondeur pour nuancer le propos; mais dans un premier temps, elle servira à montrer que les paysagistes ont plutôt intérêt à ne pas manipuler la notion de paysage urbain. Car celle-ci semble cantonner leur pratique au seul domaine de l'aménagement des lieux (à un " art urbain » en quelque sorte), quand il s'agit plutôt de mettre en relief des compétences spécifiques en matière d'aménagement à l'échelle du territoire. L'architecte paysagiste est ainsi censé prendre en compte (au-delà de l'aménagement lui-même) toutes les stratégies territoriales des acteurs en présence, et toutes les échelles dans lesquelles celles-ci se développent; il revendique ainsi l'aménagement non seulement des lieux mais du territoire dans son ensemble. Il se place directement en complémentarité (ou en concurrence parfois) avec l'architecte urbaniste; l'atout majeur du paysagiste, c'est l'image harmonieuse de la nature que l'on prête implicitement au «beau » paysage, modèle de cohérence sociale et de cohérence spatiale.

Dans ces conditions, on comprend pourquoi l'emploi de l'adjectif « urbain », accolé au mot "paysage ", ne peut servir qu'à désigner le cadre spatial, restreint, de quelques interventions d'aménagement parfois prestigieuses; cadre que l'architecte paysagiste entend bien étendre au-delà des seuls lieux intéressés...

Le projet de paysage, une façon d'accommoder l'espace urbain aux conventions naturalistes

10 En France (selon l'hypothèse posée) le mot " paysage " évoquerait avant tout l'image d'un monde rural traditionnel (la campagne et sa nature domestiquée), ou celle de sites dits «naturels» (bien que cette idée soit plus récente); milieux qu'il est convenu d'opposer à l'urbain, et même au « rurbain » contemporain. Pour oser parler du milieu urbain en termes de paysage, il manquerait ainsi (selon le philosophe A. Roger) un modèle culturel sur lequel notre regard pourrait s'appuyer :

Nous serions, devant nos villes et même nos campagnes, dans le même dénuement perceptif (esthétique) qu'un homme du XviI ${ }^{\mathrm{e}}$ siècle face à la mer et à la montagne. C'est un "affreux pays ", qui ne suscite que la répulsion. [...] Pour l'heure, nous nous complaisons dans la crise, mais c'est peut-être de cette délectation critique que sortiront les modèles de demain ${ }^{15}$.

11 De tels modèles esthétiques existent-ils déjà ? Il pourraient permettre de dépasser la répulsion paysagère (mais également la seule fascination) qu'exercent les périphéries urbaines réputées chaotiques. Certains travaux photographiques ont mis "en cadre " ces ambiances critiques de l'urbanisation; ils sont aujourd'hui reconnus tout autant pour ce qu'ils montrent que pour la façon dont ils le montrent ${ }^{16}$. La chose urbaine ellemême, parcourue ou visitée (en accord avec les représentations qui en sont faites), devient la source d'un "vécu» paysager. Le travail artistique peut relater au départ tout autre chose que le simple plaisir d'être là, il n'empêche qu'il en provoque ensuite le désir ${ }^{17}$ : et ce désir de regarder l'urbain "à la manière de » induit une "mise en paysage » de la ville, qui prend alors valeur de modèle.

12 Bien sûr, pour qu'un tel renouvellement du regard soit possible, il doit pouvoir s'élaborer sur la base d'un consensus assez large ${ }^{18}$. Ainsi parler du paysage urbain comme d'un genre de paysage (et non plus comme d'un style d'aménagement, d'une ambiance) oblige à se poser la question du seuil à partir duquel on peut "légitimement" parler de lui comme d'un paysage : affaire de notoriété esthétique (nombre de connaisseurs), mais aussi (historiquement) capacité à refléter une certaine 
sensibilité culturelle à l'espace. Or, cette valeur intrinsèque est aujourd'hui volontiers attribuée à des espaces ruraux ou en tout cas "marqués " par les éléments naturels (comme semblent le suggérer les récents «théoriciens du paysage »). L'expérience de l'espace urbain contemporain bouleverse tant les critères de cette sensibilité (pour les habitants comme pour les professionnels de l'aménagement) qu'une image urbaine du paysage reste difficilement envisageable. Si aujourd'hui les paysagistes évitent de parler de " paysage urbain ", c'est également parce que cette expression ne permet pas (encore) de rendre compte d'une expérience esthétique consensuelle de l'espace, condition indispensable à la bonne compréhension des projets de paysage.

Cette convention "naturaliste " qui détermine les paysages, liée à une approche culturelle telle que la conçoivent A. Roger et A. Cauquelin, conditionnerait des vécus paysagers engagés chaque fois dans une relation à la nature. Cette relation semble implicite dans le projet de paysage. Ainsi l'art des jardins (et des parcs) supplée en quelque sorte à l'inadéquation spatiale de l'urbain comme représentation sociale et culturelle de la nature; il s'agit de recréer des ambiances ou des ordonnancements comme autant d'avatars de cette nature pittoresque dont nous sommes empreints :

[...] pensant que ce jardin disait la campagne qui disait le paysage qui disait la nature, et trouvant dans cette entrée démultipliée la révélation $\mathrm{du}$ « beau naturel». Comment aurais-je pu l'approcher autrement que par le tableau cadré d'un jardin composé, par l'artifice de sa disposition parfaite ${ }^{19}$ ?

[...] le paysage trouve à travers l'image du jardin la valeur d'une signification propre, et la figure du jardin engage, par le microcosme qu'elle représente, le retour à une échelle de travail adéquate et correcte, permettant de retrouver la valeur perdue du paysage, le sens de l'espace extérieur ${ }^{20}$.

La " révélation » de la nature repose sur une ouverture visuelle et symbolique des lieux urbains vers un extérieur qui excède le milieu urbain ${ }^{21}$. Cette relation s'établit au moyen de dispositifs propres à l'art paysagiste, elle en définit peut-être même l'essence. Il est encore difficilement concevable en architecture des jardins de s'affranchir de ces artifices naturels que sont, par exemple, les végétaux, sauf à prétendre au seul "aménagement» urbain. En outre, pour que le regard trouve ses repères et reconnaisse quelque qualité paysagère à l'espace qui l'entoure, il faut souvent que celui-ci se présente selon les canons hérités historiquement de la vision perspective de la Renaissance ${ }^{22}$ : une étendue fuyante, un horizon. M. Corajoud ${ }^{23}$ ne dit pas autre chose $^{24}$ :

Même dans les endroits peu accidentés il y a toujours quelque chose à mettre en exergue. Une lisière, un boqueteau, une petite dépression, la rive plantée d'un ruisseau... On peut toujours récupérer quelque chose. Un des problèmes importants de la ville contemporaine me semble être son obstruction et de ce fait, sa désorientation. L'accumulation non réglée des constructions dans la ville actuelle est telle que tous les horizons se ferment. Il n'y a plus de lointains, plus de paysages, ou du moins plus cette forme de paysage que crée le rapport intelligible entre le grand territoire et les divers agencements des hommes qui l'habitent ${ }^{25}$.

La compétence et la spécificité des architectes paysagistes est ainsi de pouvoir mettre en place une stratégie, capable de faire fonctionner des dispositifs spatiaux selon plusieurs échelles de perception, afin de dépasser la seule dimension locale de l'intervention, d'en démultiplier l'espace restreint. Par ces opérations de réarticulation des lieux, par ce jeu d'imbrication de différents espaces (définis par la peinture), les paysagistes sont donc à même (et ils le revendiquent) de lutter contre cette confusion des échelles dans laquelle nous baigne le milieu urbain ${ }^{26}$. 
Le paysage et l'urbain : vers un rapport d'inclusion réciproque refermer sur le microcosme de l'intervention quand le chaos règne alentour, c'est de se contenter de la description d'une ambiance quand en réalité les lieux distants en affectent également la perception. symboliquement) les lieux urbains à ces " grands " paysages censés excéder (et parfois contenir) les villes. Pour cela, les paysagistes ont appris à articuler entre elles les diverses dimensions des espaces urbains (du microscopique au macroscopique), afin de mettre en œuvre les dispositifs nécessaires à une élaboration «in vitro" de l'expérience paysagère conventionnelle (de plus en plus difficile il est vrai à connaître in vivo). Ce qu'on attend des paysagistes, c'est en quelque sorte de pouvoir reproduire, en milieu urbain, l'expérience nostalgique d'un rapport à l'espace rural. Et que cette expérience soit relatée comme positive ou bien critique, elle s'appuie (à défaut d'illusions) sur des allusions aux milieux «naturels», et aux espaces qui les contiennent : les paysages ainsi "projetés » tiennent lieu de références " exotiques », car toujours situées au-delà l'espace urbain (espace désigné de l'artificiel).

Comme le montre A. Cauquelin, «le paysage s'identifie à la nature ${ }^{27}$ », et le projet de paysage traite avant tout de la possibilité ou de l'impossibilité de cette représentation :

Il y a donc une double porte pour donner accès aux paysagistes contemporains : ils se trouvent en effet situés - et tiraillés - entre deux termes : l'un est le versant « nature-nature » qui incitera à s'approcher, pour la dire, au plus près de la grande Nature ; l'autre prendra, à l'inverse, une distance remarquée avec cette nature, en assignant au paysage la fonction de la voiler, d'en rendre le concept ambigu, de montrer à quelle construction mentale sa perception correspond. Mais aucun de ces versants n'excluent l'autre. [...] Double face d'une interdiction: - d'une part le paysage "interdit » la nature, et d'autre part un commentaire infini force cette interdiction à paraître comme l'essence naturelle du paysage... ${ }^{28}$

Selon cette logique, les paysages en ville ne seraient évoqués qu'à travers des allusions à l'environnement géophysique (milieu «naturel» qui englobe les agglomérations, grand paysage que le regard ne peut embrasser qu'en s'échappant de l'urbain, lors de contemplations panoramiques ou aériennes). Ce paradigme «naturalisant ${ }^{29}$ » est la principale raison à réfuter la pertinence d'un modèle urbain de paysage. Comme cela a été montré plus haut, l'idée qu'un paysage puisse être urbain n'est souvent tolérée que dans la mesure où celui-ci préexiste à des projets de strict "aménagement ", en étant ainsi limité à la grandeur perceptible d'un quartier. Tandis que le projet de "paysage " vient au contraire affirmer la présence d'un espace "autre ", naturel ; celui-ci, non perçu auparavant, vient alors appuyer l'espace urbain; la présence du végétal (jardins, alignements), la révélation de la topographie (belvédères, etc.), et la possibilité de nouvelles pratiques de plein air (aires de jeu...) se combinent alors selon des logiques très géométriques d'emboîtement scalaire, de rapports d'échelle.

La seule dimension plastique de l'aménagement des lieux n'y suffit pas. Mais force est de constater que, de plus en plus souvent, la dimension végétale elle aussi ne suffit plus.

21 À ce titre, la démarche du paysagiste M. Desvigne apparaît comme une tentative avouée de renouveler à la fois la ville et la nature :

J'ai quarante-cinq ans. Je suis paysagiste depuis vingt ans. Suis-je urbaniste? Je

déteste parfois et encore la ville que nous construisons. Je ne goûte pas toujours

l'élégance de ses tracés ni de ses espaces publics et je m'ennuie dans ses jardins. 
J'arpente, je repère, j'invente des sortes de géographies, reliques ou accidentelles [...], pour tisser avec patience de vastes paysages dont j'ai la conviction qu'ils sont un espoir et une contribution à la transformation nécessaire de la ville contemporaine ${ }^{30}$. lesquels on tente parfois de donner un sens à certains quartiers périphériques. Je ne
m'intéresse à ces espaces que lorsqu'ils parviennent à s'ancrer dans un territoire ou un paysage $e^{31}$.

Militant pour un changement d'échelle d'observation de l'aménagement en ville, M. Desvigne utilise ainsi fréquemment la photographie aérienne dans ses projets de paysage. Il dit d'ailleurs vouloir inventer des « géographies » : il s'intéresse pour cela à la dimension territoriale $d u$ projet d'aménagement. Dans ces projets, les agglomérations urbaines sont d'abord déchiffrées globalement, au moyen de la carte, où les villes et les campagnes se rejoignent indifféremment dans les deux mêmes dimensions. Les «territoires» identifiés par M. Desvigne désignent pourtant, implicitement, des espaces «naturels ». Cependant, à la différence des représentations paysagistes conventionnelles, ceux-ci ne sont pas donnés à voir d'emblée comme des étendues physiques. Il est ainsi nécessaire de recourir à des représentations savantes pour en rendre compte (découpages graphiques, schémas, etc.). continuités spatiales de ce rapport urbain à la nature, d'en proposer une forme de réalité. Pour cela, M. Desvigne va même jusqu'à reconstituer (à l'occasion de son projet pour la péninsule de Greenwich à Londres) une «nouvelle nature » :

Il fallait inventer une texture qui donne des qualités à ce terrain vague, qui puisse le rendre habitable, c'est-à-dire qui protège du vent, qui cadre les vues, qui oriente... [...] une «nature intermédiaire »: un milieu vivant à l'échelle géographique, qui qualifie un territoire avec lequel il faudra composer plus tard, pour construire une ville dont la préfiguration apparaît prématurée ${ }^{32}$.

Le paysage mis en jeu par le projet (implicitement " paysage qui dit la nature », comme pour la plupart des paysagistes) se réfère alors directement à une forme de nature «urbaine» (reconstituée ici d'un seul tenant), mais qui peut tout aussi bien être interstitielle (comme lors des expériences menées par M. Desvigne sur les lieux délaissés de Boston $\left.^{33}\right)$.

Dans le projet "Lyon Confluence ", le paysagiste explique de même qu'il refuse de traiter la ville d'un bloc, comme avec une composition traditionnelle en plan de masse $^{34}$. La mise en paysage du territoire consistera ici, comme à Londres, à élaborer des stratégies hypothétiques sur trente ans, aptes à être remises en question au fur et à mesure de la métamorphose relativement imprévisible du quartier.

Tout est fait pour que la (re)constitution de l'espace naturel serve de guide à celle de l'espace urbain. M. Desvigne anticipe ainsi une ville qui se définira dans ses rapports ultérieurs à cette nature adjacente. Il recourt donc lui aussi au paysage (par le projet) comme à ce qui permet de dépasser la fermeture apparente de l'espace urbain: non plus seulement par l'approche scalaire et topographique du site (comme avec l'horizon de M. Corajoud), mais par la saisie des différentes temporalités à l'œuvre dans les processus décisionnels de l'aménagement urbain.

Toutefois, si l'aménagement semble ici réalisé antérieurement à toute activité, à toute pratique apparente, il relève encore très largement d'un rapport utopique entre une 
société urbaine et une nature intangible. Ce rapport est ici subordonné à toute une stratégie territoriale, destinée à définir les contours d'un espace naturel quand celui-ci n'est pas paré de ses atours ruraux conventionnels. Il s'agit essentiellement de définir des structures paysagères de grande ampleur, comme pour les rives de la Garonne :

L'esthétique développée a vocation à s'étendre à l'échelle du territoire. Nous proposons de vastes continuités géographiques, longeant le fleuve, se substituant à de grandes infrastructures, s'immisçant dans les quartiers selon d'anciens tracés hydrauliques. Nous définissons les dimensions nécessaires à ce paysage comme préalable aux modifications des règles de constructibilitée ${ }^{35}$.

L'espace interscalaire (mais qui permet la lecture d'un paysage et que M. Desvigne identifie ici comme une sorte d'infrastructure) est un présupposé du projet, ou en est une des résultantes ${ }^{36}$. Le projet de paysage parvient ainsi à produire l'idée d'un genre de paysage spécifiquement urbain : l'intervention (qui se situe le plus souvent dans des agglomérations déjà bien présentes) est alors l'image inversée ("à rebours») des systèmes de parcs réalisés par Olmsted, auquel M. Desvigne fait explicitement référence :

Si les systèmes de parcs américains du XIXe siècle ont servi parfois de structure à la croissance des villes, leur typologie est transposable, à rebours, pour constituer une structure aux étalements urbains contemporains. Les vestiges de la géographie, les faisceaux d'infrastructures et les sites industriels sont les lieux possibles de cette reconquête ${ }^{37}$.

M. Desvigne se sert des réalisations d'olmsted non pas tellement comme des références théoriques, mais de façon plus pragmatique comme des réalités à atteindre : tandis que les parcs urbains américains étaient réalisés dans l'attente du développement actuel qui les a submergés, l'espace urbain aujourd'hui « attendrait» le développement de ces grandes « structures paysagères » qui viendraient l'ordonner. Par cette réappropriation végétale de l'espace interstitiel, $M$. Desvigne renouvelle ainsi les références urbaines aux espaces naturels ${ }^{38}$. Il milite pour la reconnaissance d'une "nature intermédiaire ", digne d'être vécue pour elle-même, et non plus comme reproduction nostalgique. Il montre que la nature n'est jamais complètement absente des sites urbains, même les plus ingrats, et parvient ainsi à définir des paysages qui se réfèrent désormais aux agglomérations elles-mêmes, et non plus seulement à leur périphérie agricole (il est vrai que la proximité spatiale de celle-ci relève souvent, aujourd'hui, de l'abstraction).

31 Toutefois (et malgré leur dimension fractale qui perturbe les rapports d'échelle habituels), les espaces que M. Desvigne met en évidence restent empreints d'une vision paysagère culturellement conventionnelle de la ville (que donnaient aussi les systèmes de parcs du XIx ${ }^{e}$ siècle) : certes, les rapports des citadins à la nature urbaine sont ici renouvelés, mais le projet de paysage en reste dépendant. Il en suppose la nécessité. Le désir d'un paysage (comme relation symbolique à ce qui nous entoure) ne peut-il être satisfait qu'à travers la découverte et la révélation, dans l'espace urbain, d'un espace naturel qui le dédouble?

Comme le suggère A. Roger ${ }^{39}$, ce paradigme "naturalisant» des représentations urbaines du projet de paysage sera peut-être un jour remis en cause, à la faveur d'un vécu paysager du milieu urbain qui ne soit plus exclusivement associé à la recherche d'un succédané de paysage rural.

Le site : une reconfiguration de l'espace urbain comme premier désir de paysage

Le milieu urbain semble condamné par le projet de paysage à ne pouvoir qu'évoquer des genres de paysage préexistants (ceux du littoral pour un bord de fleuve, etc.). Pour 
les paysagistes, la tentation est grande également de réduire l'existence des paysages urbains à celle de simples ambiances urbaines, à de simples décors.

M. Desvigne (en cherchant à assimiler une partie de l'espace urbain à un espace naturel) remet en cause cette tendance à l'exotisme périphérique des références paysagères en milieu urbain : le projet de paysage peut tout aussi bien se référer à une nature urbaine ; le paysagiste opère alors de manière réflexive : les dispositifs mis en place par le projet pour révéler cette nouvelle nature sont dirigés cette fois vers le site lui-même, vers sa (re)constitution ${ }^{40}$. Cette idée d'une nature urbaine valorisante permet ainsi de concilier l'urbain avec une pensée somme toute assez conventionnelle du projet de paysage: car «le» paysage (comme mode de voir, comme moyen d'appréhender une certaine réalité spatiale à travers un rapport aux processus naturels) s'identifie encore une fois la nature, fut-elle fabriquée par la ville ${ }^{41}$. Le paysagiste se garde bien de parler à ce propos de paysages urbains... préférant parler alors d'architecture du territoire ${ }^{42}$, et insistant ainsi sur le travail de reconfiguration de l'espace plutôt que sur celui de l'expérience paysagère.

Pourquoi alors ne pas accepter de qualifier la forme vécue de cette expérience, propre au milieu urbain, de paysage urbain?

Cela irait vraisemblablement trop loin; car il ne s'agit plus seulement de reconnaître un genre de paysage qui décrirait la ville comme il décrit la campagne. Il s'agit de mettre en place une manière de voir qui entre en concurrence avec «le » paysage luimême : qui suppose une autre façon d'appréhender la réalité, bien au-delà des seules préoccupations naturalisantes. Cette autre vision serait plus attentive aux conditions initiales de l'expérience paysagère (au paysage comme expérience de la réalité) qu'aux représentations conventionnelles de celle-ci. Cela impliquerait donc de parler de l'introversion des lieux urbains, de leur repli, tandis que les paysagistes n'ont de cesse de vouloir les connecter à un ailleurs, privilégiant à l'expérience vécue de l'espace urbain la mise en place d'une présence symbolique de la nature. La logique de mise en espace du projet de paysage n'est pas directement mise en cause : elle s'élargit au contraire vers d'autres pratiques possibles des lieux aménagés, vers d'autres paradigmes.

Car il est impensable que les architectes paysagistes, même s'ils en parlent peu, ne mettent pas sans cesse à l'épreuve leurs manières de voir tout autant que leurs manières de faire. Leur présence dans le domaine de l'urbanisme l'atteste bien : il s'agit d'abord de faire partager par le projet d'aménagement une certaine forme de réalité, basées sur des expériences bien localisées: situées. D'où ce sentiment qu'avant tout projet de paysage, au commencement de toute tentative de mise en rapport des lieux et des espaces, avant tout essai de spatialisation, il y aurait un désir, vécu, de paysage. Et si le paysagiste oriente par la suite sa recherche du côté d'un mode de représentation spatial qui lui est familier, c'est tout autant par déformation professionnelle que par habitude culturelle. Ce choix (qui exclut le paysage urbain pour les raisons suggérées ci-dessus) n'est pourtant qu'une manière parmi d'autres d'exprimer cette première expérience paysagère ressentie sur le «site »: d'autres disciplines en donneront des traductions forts différentes (le cinéma, la photographie, la littérature, etc.). La transformation de ce premier "vécu " paysager en représentation paysagère potentielle est donc une étape importante et marquante du projet de paysage.

Il est ainsi fréquent que les paysagistes (mais les architectes également) relatent cette expérience particulière de la première saisie d'un site (de son "invention", au sens 
archéologique). Bernard Lassus insiste à ce propos sur la nécessité, pour le paysagiste, de se placer en situation d'attention "flottante » :

S'imprégner, au cours de longues visites à diverses heures et par tous les temps, du site et de ses alentours, «faire l'éponge » de sol à ciel presque jusqu'à l'ennui. Puis chercher des points de vue préférentiels, déceler les micro-paysages et les perspectives qui les lient, repérer puis tester les échelles visuelles et tactiles... tout en consultant ses mémoires, lieux-dits, contes et légendes locales, les histoires, l'Histoire ${ }^{43}$.

Ici, le cheminement par la marche à pied est préconisé comme une façon de "voir » le site, de le connaître pour mieux pouvoir le reconnaître, malgré la réduction géométrique $\mathrm{du}$ dessin de projet. C'est une manière d'acquérir un savoir, la connaissance du paysagiste étant tout entière tendue vers le devenir du site, vers sa mise en espace. Toutefois, comme le souligne $\mathrm{M}$. Corajoud, c'est la "première seconde » qui est décisive, parce que c'est elle qui va formater le désir de paysage selon la visée spatialisante du projet :

Ce qui fait le regard d'un paysagiste maître d'œuvre sur un site par rapport au regard de quelqu'un d'autre, c'est qu'il possède la faculté d'anticiper la transformation du site. Son regard met en jeu à la première seconde tout un savoir et toute une expérience sur la modification de l'espace. Le regard du scientifique sur le même espace n'anticipe rien, il est riche d'enseignements mais il ne permet pas d'apercevoir la modification de cet espace. Le maître d'œuvre, lui, met en jeu parallèlement à la sensibilité du regard tous les mécanismes de la transformation : tout un savoir parallèle. Bien sûr : le site, le site, le site; mais ce site ne dit rien de sa transformation si la personne qui le regarde n'est pas elle-même capable de l'anticiper ${ }^{44}$.

Cette aisance du paysagiste à reconnaître ainsi les potentialités spatiales du site est cependant toute relative, car il s'agit finalement pour lui de s'extraire très vite d'une perception quotidienne de l'espace et des lieux, afin de ne pas perdre le bénéfice de représentations plus « métrisables »:

[...] comment œuvrer sur des sites où nous sommes étrangers ? Il y a la nécessité d'accumuler du sensible et de la connaissance très vite pour pouvoir œuvrer correctement sur l'espace. Il y a toujours une grande boulimie au début d'un projet car il faut absolument acquérir de la connaissance pour pouvoir agir sur un lieu. [...] Quand tu restes sur un site tu n'es plus capable de prendre une décision, tu es enfoui dans la masse des données. Il faut accepter de t'en dissocier. [...] Tu reviens à ta planche, là où tu peux opérer d'une façon abstraite sur le site. Il est vrai que nous avons une difficulté notable: tout ce que le paysagiste regarde est fuyant, gauche, indiscipliné, circonstanciel. La géométrie est intéressante, elle nous permet d'attraper un peu tout ça, de regarder quelle est la plus grande dimension de ceci, de voir comment on peut le comprendre, en partie, l'asservir à une règle plus élémentaire ${ }^{45}$ ?

Les conditions initiales de l'expérience paysagère urbaine se dissolvent dans le projet de paysage. L'abstraction qui seule permet de relier l'urbain au naturel fausse le regard, qui d'expérimental devient conventionnel. On voit donc dans quelle mesure la quotidienneté nuit au projet, car comme forme de réalité elle concurrence celle de la représentation paysagère du site par le dessin (alors liée à l'espace géométrique, bien plus consensuel). C'est donc à la seule prise de contact avec le site qu'il faudrait s'intéresser, pour y déceler la capacité du milieu urbain à évoquer des réalités paysagères qui soient finalement indépendantes des conventions du projet. 
La découverte du «terrain » fait d'ailleurs souvent l'objet de récits, au même titre que les réalisations ${ }^{46}$; et il est possible d'y noter une certaine fascination pour ce qui n'est encore, le plus souvent, qu'un délaissé urbain. Souvent, la description de la découverte du site est l'occasion de "révéler » un paysage encore introverti ; cette révélation s'exprime alors souvent sous la forme d'un monologue intérieur, tel qu'il serait tenu hors-champ ou plutôt hors-site. La voix « off » du paysagiste, en relatant ce premier désir d'un paysage (cette première "vision») donne ainsi le sentiment que l'intervention se sera plus tard inscrite dans ce contexte antérieur plus local, paysage « cosmophanique ${ }^{47}$ » chargé de supporter la fiction à venir.

Cette voix d'outre-monde (comme celle du jeune Desvigne, qui se fabrique, à pied, un paysage du quotidien ${ }^{48}$ ) se place en quelque sorte en dehors de l'intervention proprement dite : elle définit une sorte de paysage d'avant projet. Ce qui révèle toute l'ambiguïté à parler de "projet de paysage »: en fin de compte, les architectes paysagistes ne «créent » pas de paysages; ils communiquent à d'autres le désir que ceux-ci puissent exister, par des métaphores spatiales. Ce sont des paysages avant tout fictionnels. Et l'on voit bien alors que le désir de paysage, qui naît de la reconnaissance du site, n'est pas synonyme d'exigence de nature ; c'est peut-être même cette absence qui finalement rend nécessaire la fiction de paysages qui naîtraient de l'urbain, paysages-urbain qui se dissoudraient ensuite dans les évocations trop souvent conventionnelles des lieux paysagés.

4 Toujours est-il que le projet des architectes paysagistes est d'abord de faire « apparaître » ces paysages, en délimitant des sites (en définissant des points de vue, des ouvertures, des connexions possibles entre les lieux et ce qui les excèdent), quand le simple aménagement architectural des lieux urbains s'arrête lui à leur fonctionnalité et à leur mise en scène. Cette notion de site peut donc permettre de dépasser la limitation morphologique attribuée abusivement au paysage urbain puisque les paysages «avant projet " ne renvoient en première instance qu'à des situations purement urbaines.

4 Le site mélange tout à la fois l'histoire d'un lieu, sa topographie, les vues qu'il rend possible vers l'extérieur mais également celles qui le révèlent... sans pour autant mettre en jeu un quelconque rapport à des espaces naturels. Ainsi, dans son essai Le site et le paysage $^{49}$, A. Cauquelin a montré que la notion de site recouvre en fait plusieurs types de spatialités, et correspond ainsi à une spatialité « hybride » qui en articulerait deux autres : celle, intime et sensible, qui fait la singularité des lieux ; et celle, plus abstraite et hiérarchisée, de la représentation cartographique ${ }^{50}$. Suivant cette définition, le site permettrait alors le développement d'un dispositif capable d'articuler non plus seulement les différentes échelles spatiales (le lieu et son point de vue sur l'étendue), mais également les différentes natures de spatialité que met en présence l'expérience réelle aujourd'hui.

A. Cauquelin montre à ce propos que si le site définit effectivement un point de vue sur le monde, il est avant tout «ce qui n'est pas vu, mais qui donne à voir. [...] il rend perceptible ce qui l'entoure ${ }^{51} »$. Il peut donc également être appréhendé en termes de réseau (l'auteur prend l'exemple du site web), de cheminement, de mise en relation des lieux, et non plus seulement en termes de position géographique (situm) :

Ainsi peut-on remarquer que d'un côté « site » offre dans son usage tout un monde de stabilité, de protection ; il permet de se positionner soi-même et de positionner tout élément individuel par rapport aux autres. [...] D'un autre côté, en revanche, ce 
sont des espaces éphémères, des occasions, des points de vue changeants qui sont évoqués. Site se rapproche alors de la vision contemporaine d'un monde où les choses et les gens sont en mouvement ${ }^{52}$. Déjà saisis par l'intuition du paysagiste (qui les "naturalise »), ce sont eux qui provoquent finalement son désir de prolonger le site (devenu lieu pratiqué) vers d'autres horizons.

\section{Conclusion}

Chaque ville a des possibilités spécifiques de constituer son paysage, nourri de formes et de rythmes passés, et d'autres possibilités présentes, en devenir. Les réalités d'une ville ne se rendent accessibles qu'au travers de problématisations. Or poser un problème ne se fait qu'à partir de pratiques; pratique du voir, pratiques $\mathrm{du}$ faire et pratiques du dire ne sont pas identiques. Parler du paysage ne reproduira jamais ce qu'on en voit, comment on le fait ou ce qu'on y fait. Montrer des images ne suffira pas à témoigner de ce qu'on en dit, ou comment on l'a pensé ${ }^{54}$.

Beaucoup de sites urbains ne sont plus que des lieux paysagés, par la force des projets et des décisions politiques; mais il en existe une infinité d'autres, qui s'inventent au jour le jour par la seule pratique des agglomérations, à travers les topologies qu'elles instaurent. Ainsi la vie urbaine ne se résume pas à quelques instants de récréation ou de contemplation: manquerait-il la plupart du temps au paysagiste l'expérience quotidienne des sites qu'il aménage? C'est pourtant selon cette forme de réalité que s'établit le lien, vécu, entre les diverses spatialités et temporalités des lieux agglomérés, et les genres de paysages qu'ils suscitent, sans recourir à l'architecture. Cette " cécité » $\mathrm{du}$ quotidien explique peut-être pourquoi, aux yeux du paysagiste, il est si important de 
définir des stratégies pour conquérir toujours plus d'espace naturel: car il s'agit d'ancrer, dans le temps du projet et de ses conventions, ces incertitudes spatiales qui sont pourtant le lot de la vie urbaine. Le paysagiste peut alors y prendre ses habitudes, sur le papier du moins, et par le biais d'une discipline quotidienne (la pratique professionnelle remplace alors la pratique d'usage).

Rompant avec cette conception paysagiste, l'enjeu que portent les paysages urbains ne se situe pas dans cette multiplication d'horizons distants qui étendent les lieux urbains vers une nature hypothétique. Bien au contraire, car les pratiques de déplacement révèlent aujourd'hui une autre forme d'extension des sites, une autre topologie, plus réticulaire. Les agglomérations contemporaines se composent de constellations de lieux et de situations, dont le parcours en définit la substance spatiale ${ }^{55}:$ relation nécessaire et suffisante à une expérience paysagère urbaine qui peut désormais s'affranchir de la médiation d'un espace naturel et de ses représentations conventionnelles.

Ce n'est pas pour autant que l'on doit se représenter de facto l'urbain par des paysages, le premier tenant lieu de "nouvelle nature "; car cela relève de logiques et de stratégies d'autant plus complexes que notre rapport à celle-ci s'établit de manière toujours moins explicite. L'enjeu est ici plus opératoire ; il s'agit de reconnaître que des représentations caractéristiques de l'espace urbain peuvent tenir lieu de paysage: c'est-à-dire non pas que le paysage puisse devenir urbain, mais que les pratiques urbaines définissent, pour elles-mêmes, des formes de paysage.

\section{NOTES}

1. M. Desvigne, Recherche pour une esthétique de la transformation des territoires, Note à l'attention des membres du jury, Grand prix de l'urbanisme 2003, 2003. C'est nous qui soulignons.

2. Selon que ces représentations décrivent des paysages, ou qu'elles se rapportent à eux.

3. Un ouvrage collectif déjà ancien (A. Berque et al., Cinq propositions pour une théorie du paysage, Seyssel, Champ Vallon, 1994) précise certaines de ces postures, pour lesquelles « le paysage est une entité relative et dynamique, où nature et société, regard et environnement sont en constante interaction » (p. 6).

4. A. Roger, Court traité du paysage, Paris, Gallimard, 1997, p. 126 et 130.

5. Article « Paysage », in P. Robert et al., Le nouveau petit Robert : dictionnaire alphabétique et analogique de la langue française, Paris, Dictionnaire Le Robert, 2001. Nous soulignons.

6. L'auteur américain J. B. Jackson s'élève d'ailleurs dès 1984 contre cet usage qui s'appliquerait, de la même façon, au mot landscape ; toutefois, l'étymologie anglosaxonne semble plus lisible (land: espace défini, scape : forme organisée), tandis que celle plus latine de « paysage » ne permet pas de retrouver de façon aussi évidente les racines territoriales du mot (suffisamment évidente en tout cas pour influencer le sens commun). Ainsi, pour J. B. Jackson, le paysage serait avant tout une « composition d'espaces faits par l'homme sur la Terre ». Voir J. B. Jackson, À la découverte du paysage 
vernaculaire, préface de J.-M. Besse et G. A. Tiberghien, trad. X. Carrère, Arles, Rennes, Actes sud-ENSP, 2003, p. 45-57 (édition originale : Discovering the vernacular landscape, New Haven, Yale University Press, 1984). Se reporter notamment à la préface française de cet ouvrage.

7. En effet, dans le même temps, les géographes travaillant sur les « représentations » de l'espace ont a contrario fortement investi le champ de l'urbain, en délaissant alors l'usage du mot paysage, peut-être trop lourd de sens en géographie. Pour resituer ces débats, sur la " théorie du paysage » d'un côté, et la " géographie représentationniste » de l'autre, voir S. Bonin, « Au-delà de la représentation, le paysage », Strates, matériaux pour la recherche en sciences sociales, $n^{\circ} 11,2004$, p. 13-26.

8. Que ce milieu soit décliné à l'échelle du quartier, voire de la rue, jusqu'à l'agglomération en passant par les villes (2e édition ; édition originale : Plon, 1989).

9. A. Cauquelin, L'invention du paysage, Paris, PUF, 2000, p. 31

10. Avec deux ouvrages qui « encadrent » les débats des années 1990 : L'invention du paysage, en 1989, et A. Cauquelin, Le site et le paysage, Paris, PUF, 2002.

11. J. Coulon, «Qu'est-ce qu'un paysagiste? ", Revue-Annuaire des anciens élèves de l'ENSP de Versailles, 2000, p. XIX (cité par M. Audouy, « Du jardin public au paysage », Études normandes, $\left.n^{\circ} 3,2003\right)$.

12. Anthos, $n^{\circ} 02 / 2003$ (Paysages urbains), 2003 ; Quaderns d'arquitectura $i$ urbanisme, $\mathrm{n}^{\circ} 228$ (Paisatges urbans), 2001 ; Topos, $\mathrm{n}^{\circ} 23$ (Landschaften der Ereignisse-Landscapes for events), 1998.

13. Ici, l'École nationale supérieure du paysage, et l'École d'architecture de Paris-la Villette.

14. Le Centre de documentation de l'urbanisme a ainsi commandé, il y a quelques années, une note de synthèse, rédigée avec le concours du paysagiste $P$. Girardin, qui aborde les rapports entre paysage et aménagement (Atelier Pierre Girardin et Aménagement et nature, Paysage et aménagement urbain. Note de synthèse, Paris, Ministère de l'Équipement, des Transports et du Logement, Centre de documentation de l'urbanisme, 2001, http://www.urbanisme.equipement.gouv.fr/cdu/accueil/ bibliographies/paysamenag/amenat.htm. Ce document plaide pour le recours aux architectes paysagistes dans le projet urbain : leur capacité à appréhender la complexité urbaine serait justifiée par leur compétence à maîtriser la complexité des interventions paysagères (le paysage étant lui-même un objet théorique complexe, qui nécessite de penser l'espace suivant plusieurs échelles d'intervention).

15. A. Roger, Court traité du paysage, Paris, Gallimard, 1997, p. 113.

16. Les architectes se sont d'ailleurs pris à ce jeu. Ainsi les photographies prises par

R. Koolhaas (notamment celles qui sont reproduites en guise de prologue dans son fameux article "The Generic City ») illustrent-elles un désir d'instaurer une vision paysagère avant tout urbaine, qui serait liée au déplacement... Voir R. Koolhaas et al., Small, medium, large, extra-large : Office for Metropolitan Architecture, Rem Koolhaas and Bruce Mau, Rotterdam - New York, 010 Publishers - Monacelli Press, 1995, p. 1238-1247 (trad. française : R. Koolhaas, «La ville générique », Architecture d'Aujourd'hui, n 304, 1996, p. 70-77).

17. Il s'agit du phénomène de la double articulation in visu - in situ, mis en évidence par A. Roger, et qu'il nomme « artialisation » : «Processus artistique qui transforme et embellit la nature, soit directement (in situ), soit indirectement (in visu), au moyen de modèles. » (A. Roger, « Artialisation », in A. Berque et al., Mouvance. Cinquante mots pour le paysage, Paris, Éditions de la Villette, 1999, p. 45). Un artefact (l'environnement 
urbain) peut ainsi à la longue en inspirer un autre : le regard esthétique qui, toujours selon A. Roger, instaure le « pays » en " paysage » (ou ici l'urbain en " paysage urbain » ?). L'influence peut ensuite devenir réciproque, l'esthétique influençant l'intervention concrète.

18. Le travail « paysagiste » (au sens premier du terme : celui du peintre, du « descripteur » de paysages) devient alors « artialisateur », voir infra.

19. A. Cauquelin, L'invention du paysage, Paris, PUF, 2000, p. 33.

20. L. Chenu, «Paysages », Faces, $n^{\circ} 24,1992$, p. 6-8. Nous soulignons.

21. Jusqu'au planétaire donc - avec G. Clément - ou jusqu'au souvenir des Amériques comme avec le jardin des Retours de B. Lassus, à Rochefort-sur-Mer.

22. A. Cauquelin, L'invention du paysage, Paris, PUF, 2000, p. 29-30.

23. « Paysagiste », comme il l'écrit, entre guillemets. Étudiant à l'École des arts décoratifs, il devient paysagiste en travaillant avec J. Simon, lui-même photographe de formation. Tous deux enseignent à l'École nationale supérieure du paysage.

M. Corajoud obtient le Grand prix du paysage en 1993 et le Grand prix de l'urbanisme en 2003.

24. Même si sa définition de l'horizon ne se limite pas à la seule interprétation visuelle de la ligne d'horizon...

25. M. Corajoud, « Hors champ ", Faces, $n^{\circ} 55,2004$, p. 14-17. Nous soulignons.

26. Et dont parle, par exemple, F. Choay : « Sur la nouvelle Babel s'abat une nouvelle malédiction : la confusion des échelles, qui brouille la scène urbaine et rend indiscernable la différence des enjeux et des acteurs qui s'y confrontent. Règne de l'urbain, effacement de la ville, échelle unique d'aménagement : plutôt que de nous voiler la face devant ces évidences, il conviendrait d'en tirer les conséquences » (F. Choay, «Le règne de l'urbain et la mort de la ville », in J. Dethier, A. Guiheux (dir.), La ville, art et architecture en Europe : 1870-1993, Paris, Centre Georges Pompidou, 1994, p. 34).

27. A. Cauquelin, L'invention du paysage, Paris, PUF, 2000, p. 31.

28. Ibid., p. 149.

29. Pour ne pas dire « naturaliste » : mais cette allusion serait abusive; toutefois, réalisme mis à part, l'intervention du paysagiste s'établit le plus souvent dans un rapport d'échelle qui met en exergue une (ou plusieurs) dimension naturelle dans la représentation sensitive des lieux.

30. M. Desvigne, Recherche pour une esthétique de la transformation des territoires, Note à l'attention des membres du jury, Grand prix de l'urbanisme 2003, 2003, p. 2. Nous nous référerons essentiellement, concernant les travaux de M. Desvigne et les commentaires qu'il en a fait lui-même, à cette note " introspective » qu'il a produite lors de sa candidature au Grand prix de l'urbanisme en 2003.

31. Ibid., p. 24. C'est nous qui soulignons.

32. M. Desvigne, «Le paysage, nature intermédiaire », AMC Le Moniteur Architecture, $\mathrm{n}^{\circ} 101,1999, \mathrm{p} .60$. C'est nous qui soulignons.

33. M. Desvigne, Ibidem note 30, p. 14.

34. Ibid., p. 15.

35. Ibid., p. 18. C'est nous qui soulignons.

36. «[...] nous tissons de formidables réseaux d'autoroutes et de voies ferrées, mais nous répugnons à doter la périphérie de nos villes de structures qui dépassent la distance qui sépare deux ronds points. Cette « accommodation » de l'échelle me semble être un enjeu théorique majeur de l'architecture du territoire» (ibid., p. 23). 
37. Ibid., p. 12.

38. Et rejoint ainsi des préoccupations environnementales et écologiques.

39. Voir citation, infra (« c'est peut-être de cette délectation critique que sortiront les modèles de demain »).

40. Ainsi M. Desvigne parle de « jardins-prototypes », qui lui permettent de tester à petite échelle ces dispositifs : "J'utilise ces petites réalisations pour expérimenter des dispositifs que nous avons imaginés développer pour de grands territoires (densité, gestion de la croissance...). J'envisage ensuite la transformation (souvent lointaine, hypothétique ou aléatoire) des grands sites avec la « mesure » de ces expériences et avec pour les deux échelles le même désir de « réalité » et « d'architecture » (ibid., p. 7). 41. Comme l'indique le titre de cet article: M. Desvigne, « Le paysage, nature intermédiaire ", AMC Le Moniteur Architecture, n 101, 1999, p. 60.

42. M. Desvigne, Ibidem note 30, p. 23.

43. B. Lassus, "Analyse inventive ", in A. Berque et al., Mouvance. Cinquante mots pour le paysage, Paris, Éditions de la Villette, 1999, p. 45.

44. M. Corajoud, P. Madec, « La part commune », Techniques et architecture, $\mathrm{n}^{\circ} 403,1992$, p. 74-75.

45. Ibid. Nous soulignons.

46. Cette piste restera toutefois ici exploratoire, mais elle est à poursuivre de façon plus systématique.

47. Dans son ouvrage Écoumène, le géographe A. Berque définit cinq critères qui permettent d'après lui de pouvoir parler de paysage et non pas d'un simple désir de représenter l'environnement. Quand ces conditions ne sont pas remplies, il propose d'utiliser le terme de « cosmophanie »... (A. Berque, Écoumène : introduction à l'étude des milieux humains, Paris, Belin, 2000, p. 160-161). Dans l'impossibilité encore d'être donné à voir, le désir de paysage serait ainsi, en première instance, cosmophanique?

48. Voir épigraphe.

49. A. Cauquelin, Le site et le paysage, Paris, PUF, 2002.

50. Ibid., p. 78-87.

51. Ibid., p. 27.

52. Ibid., p. 22.

53. Il est fait ici appel à la notion de « lieu pratiqué » de M. de Certeau, et qu'il nomme tout simplement « espace » : «L'espace serait au lieu ce que devient le mot quand il est parlé, c'est-à-dire quand il est saisi dans l'ambiguïté d'une effectuation, mué en un terme relevant de multiples conventions, posé comme l'acte d'un présent (ou d'un temps), et modifié par les transformations dues à des voisinages successifs. À la différence du lieu, il n'a donc ni l'univocité ni la stabilité d'un “propre”. En somme, l'espace est un lieu pratiqué. Ainsi la rue géométriquement définie par un urbanisme est transformée en espace par des marcheurs »: M. de Certeau, L'invention du quotidien. 1. Arts de faire, 1990, Paris, Gallimard, p. 172-173 (nouvelle édition par L. Giard ; édition originale : 1980).

54. M. Claramunt, $C$. Mosbach, « De loin, de près, une ville, le paysage ", Les annales de la recherche urbaine, $\mathrm{n}^{\circ} 85,1999$, p. 176.

55. Voir à ce propos : G. A. Tiberghien, J.-M. Besse, " Hodologique ", Les carnets du paysage, $\mathrm{n}^{\circ} 11,2004$, p. 6-33, pour un développement plus complet du lien entre topologie, cheminement et paysage. 


\section{RÉSUMÉS}

Diverses opérations d'urbanisme récentes sont cosignées (en France notamment) par des architectes paysagistes de renom; mais tandis que les commentateurs qualifient volontiers les quartiers d'aujourd'hui comme autant de "paysages urbains ", ces paysagistes s'en abstiennent, quand ils discourent sur leurs projets et leurs réalisations. Ce paradoxe apparent est discuté ici. Il sert de prétexte à poser certaines hypothèses sur les possibilités d'une «mise en paysage » des espaces urbains. En effet, dans le domaine du paysagisme, les représentations esthétiques de l'urbain se trouvent systématiquement associées sinon opposées à celles d'une nature toujours plus utopique et désirable (ces dernières faisant seules, par convention, "paysage »). C'est un trait que l'on retrouve aussi bien à travers les postures professionnelles qu'à la lecture des travaux entrepris dans les années 1990 en France sur les «théories du paysage». Or, les conventions «naturalistes » de la représentation paysagère ne sont pas sans conséquence sur l'expérience de la ville. Elles tendent à instaurer des relations extraordinaires, mais distantes et figées, avec une nature devenue hypothétique; tandis que les pratiques urbaines quotidiennes, par les circonvolutions des espaces-temps qu'elles induisent, appellent à une redéfinition non plus seulement topographique mais également topologique des paradigmes paysagers.

Florent Hébert, The urban landscape paradox in discourse on landscaping

Various recent operations of urban design are co-signed (in France in particular) by famous landscape architects; but while commentators qualify recent neighbourhoods as "urban landscapes", the designers don't use the same terms when talking about their projects and their works. This apparent paradox is discussed here. It is used as an opportunity to present some hypotheses around the possibility of "landscaping" of urban spaces. Indeed, in the field of landscaping, the aesthetic representations of urban state are systematically associated (if not opposite) with those of a natural state, becoming increasingly more utopian and desirable (these last being, by convention, the very "landscape"). It is a feature which can be found through the professional postures, but as well in the reading of the work undertaken in the Nineties in France about "landscape theories". However, "naturalistic" conventions for landscape representations are not inconsequential about experimenting the city; they tend to organize uncommon (but distant and fixed) links, with a nature becoming more and more hypothetical; while urban patterns and daily practices, by the circumvolutions of space-time they induce, call for a redefinition (not only topographic, but also topological) of the landscape paradigms...

INDEX

Keywords : France, landscape, urban landscape, site, landscape theory, landscaping, routine Mots-clés : paysage, paysage urbain, théorie du paysage, projet, quotidien

\section{AUTEUR}

\section{FLORENT HEBERT}

Architecte urbaniste de l'État, doctorant géographe à l'université de Paris-I (DEA option Jardins, paysages, territoires), Ladyss (Laboratoire dynamiques sociales et recomposition des espaces), CNRS, 2 rue Valette, 75005 - Paris : fl.hebert@free.fr 